\title{
Sensibilité, spécificité, valeur prédictive positive et valeur prédictive négative d'un test diagnostique
}

\author{
M.R. Nendaz, A. Perrier
}

Clinique de Médecine 1, Hôpitaux Universitaires de Genève, Suisse.

Tirés à part : M. Nendaz, Clinique de Médecine 1,

Hôpitaux Universitaires de Genève, Rue Micheli-du-Crest 24,

$\mathrm{CH}-1211$ Genève 14, Suisse.

mathieu.nendaz@hcuge.ch

Réception version princeps à la Revue : 24.09.2003.

Retour aux auteurs pour révision : 31.10.2003.

Réception $1^{\text {ère }}$ version révisée : 24.11.2003.

Acceptation définitive : 26.11.2003.
Pour étayer une hypothèse diagnostique le clinicien utilise fréquemment des symptômes et signes cliniques ainsi que des examens complémentaires. Ces tests (pour notre propos, des symptômes et signes cliniques peuvent également être considérés comme tels) devraient idéalement donner un réponse univoque à la question diagnostique : maladie ou condition présente si le test est positif, absente s'il est négatif. Malheureusement, dans la pratique, il y a peu de signes cliniques ou de tests pouvant être interprétés de façon univoque. Certains patients présentent en effet un signe ou un test positifs, sans pour autant souffrir de la maladie recherchée; d'autres, au contraire, bien que porteurs de la maladie recherchée, ne présentent pas ce signe clinique ou présentent un test négatif. Ainsi, le clinicien doit-il pouvoir disposer des caractéristiques d'un test lui permettant d'en interpréter les résultats et d'appliquer ces derniers à son patient : ce sont les qualificatifs de sensibilité, spécificité et valeurs prédictives, notions qui seront abordées dans cet article à travers un exemple.

Vous vous intéressez à la performance de l'appréciation clinique et du dosage du Peptide Natriurétique de type B (PNB) pour diagnostiquer une insuffisance cardiaque face à une dyspnée aiguë. Vous effectuez une recherche de littérature et choisissez un article de PA McCullough qui semble répondre à votre question [1]

Ce travail prospectif et multicentrique effectué en milieu d'urgence a pour objectif de déterminer les caractéristiques diagnostiques du jugement clinique des médecins recevant des patients souffrant de dyspnée aiguë de même que celles du PNB (à un seuil de $100 \mathrm{pg} / \mathrm{ml}$ ), face au diagnostic de référence effectué par deux cardiologues de manière aveugle. Cette étude inclut 1538 patients avec dyspnée aiguë, dont 722 (prévalence de $47 \%$ ) sont finalement considérés comme souffrant réellement d'insuffisance cardiaque.

Les caractéristiques diagnostiques de l'appréciation clinique et du dosage du PNB rapportées par les auteurs sont résumées dans le tableau $I$. Il est à relever que l'on considère qu'un clinicien retient le diagnostic d'insuffisance cardiaque s'il en a une certitude d'au moins $80 \%$, et que le dosage de 
Tableau I.

Résumé des caractéristiques du jugement clinique et de la mesure de PNB (à une valeur-seuil de $100 \mathrm{pg} / \mathrm{ml}$ ) pour diagnostiquer une insuffisance cardiaque chez un patient souffrant de dyspnée aiguë, rapportées par McCullough et coll. [1].

\begin{tabular}{lcc}
\hline & Valeur (\%) & IC 95 \% \\
\hline Jugement clinique & 49 & $47-52$ \\
Sensibilité & 96 & $95-97$ \\
Spécificité & 91 & $90-92$ \\
Valeur prédictive positive & 68 & $66-71$ \\
Valeur prédictive négative & & \\
PNB $\geq \mathbf{1 0 0} \mathbf{~ p g / m l}$ & 90 & $89-92$ \\
Sensibilité & 73 & $71-73$ \\
Spécificité & 75 & $72-77$ \\
Valeur prédictive positive & 89 & $88-91$ \\
Valeur prédictive négative & & \\
\hline
\end{tabular}

PNB est considéré comme positif pour une insuffisance cardiaque à partir d'une valeur de $100 \mathrm{pg} / \mathrm{ml}$.

Sur la base de ces données, on peut déduire le nombre absolu de patients de l'étude qui ont réellement une insuffisance cardiaque ou qui ne l'ont pas, ceux dont le diagnostic clinique était correct ou non, et ceux dont le PNB était positif ou négatif. On peut ainsi créer les tableaux II et III qui serviront de base à la discussion des notions de sensibilité, spécificité, valeur prédictive positive et valeur prédictive négative, en considérant le jugement clinique et le PNB comme deux tests diagnostiques indépendants.

\section{Tableau II.}

Répartition des patients en fonction de leur statut d'insuffisance cardiaque et du résultat du jugement clinique.

\begin{tabular}{lccc}
\hline & $\begin{array}{c}\text { IC réellement } \\
\text { présente }\end{array}$ & $\begin{array}{c}\text { IC réellement } \\
\text { absente }\end{array}$ & Total \\
\hline Jugement clinique + & 353 & 33 & 390 \\
Jugement clinique - & 369 & 783 & 1148 \\
Total & $722^{*}$ & 816 & $1538^{*}$ \\
\hline
\end{tabular}

*Données disponibles dans l'étude de McCullough, les autres valeurs étant déduites à partir des caractéristiques du test diagnostique fournies dans l'étude.

\section{Tableau III.}

Répartition des patients en fonction de leur statut d'insuffisance cardiaque et du résultat du dosage du PNB (une valeur-seuil de 100 $\mathrm{pg} / \mathrm{ml}$ ou plus définissant un test positif).

\begin{tabular}{lccc}
\hline & $\begin{array}{c}\text { IC réellement } \\
\text { présente }\end{array}$ & $\begin{array}{c}\text { IC réellement } \\
\text { absente }\end{array}$ & Total \\
\hline PNB + & 650 & 220 & 870 \\
PNB - & 72 & 596 & 668 \\
Total & $722^{*}$ & 816 & $1538^{*}$ \\
\hline
\end{tabular}

*Données disponibles dans l'étude de McCullough, les autres valeurs étant déduites à partir des caractéristiques du test diagnostique données dans l'étude.

\section{Sensibilité}

La sensibilité d'un test est déterminée sur une population de patients dont on sait qu'elle est porteuse de la maladie $M$ parce qu'elle a subi un test de référence. Elle est définie par la proportion ( \%) de patients qui ont la maladie recherchée et dont le test est positif, en d'autres termes par la proportion de patients malades de la maladie $\mathrm{M}$ que le test détecte correctement (vrais positifs). Par opposition, la proportion de patients porteurs de la maladie $\mathrm{M}$ que le test n'a pas identifiés sont des résultats faussement négatifs.

Appliquons ces définitions aux données du tableau II. Le jugement clinique permet d'identifier 353 patients insuffisants cardiaques sur 722 . La proportion de patients identifiés est donc de 353/722, soit $49 \%$ de tests vrais positifs. La sensibilité du jugement clinique pour le diagnostic de l'insuffisance cardiaque chez des patients se présentant avec dyspnée aiguë est donc de $49 \%$.

En effectuant les mêmes calculs sur les valeurs du tableau III, vous devriez maintenant trouver la sensibilité du dosage du PNB dans le diagnostic de l'insuffisance cardiaque (la réponse, arrondie à la décimale, se trouve dans le tableau I).

\section{Spécificité}

La spécificité d'un test est déterminée sur une population de patients dont on sait qu'elle n'est pas porteuse de la maladie $\mathrm{M}$ parce qu'elle a subi un test de référence. Elle est définie par la proportion ( \%) de patients qui n'ont pas la maladie recherchée et dont le test est négatif, en d'autres termes par la proportion de patients non malades de la maladie $\mathrm{M}$ que le test détermine correctement (vrais négatifs). Par opposition, la proportion de patients non porteurs de la maladie $\mathrm{M}$ chez qui le test est positif sont des résultats faussement positifs.

Appliquons également ces définitions aux données du tableau II. Le jugement clinique permet de classer correctement 783 patients sur 816 qui ne sont pas insuffisants cardiaques. La proportion de jugements cliniques corrects pour des patients non insuffisants cardiaques est donc de 783/816, soit $96 \%$ de tests vrais négatifs. La spécificité du jugement clinique pour le diagnostic de l'insuffisance cardiaque chez des patients se présentant avec dyspnée aiguë est donc de $96 \%$.

En effectuant les mêmes calculs sur les valeurs du tableau III, vous devriez également trouver la spécificité du dosage du PNB dans le diagnostic de l'insuffisance cardiaque (la réponse, arrondie à la décimale, se trouve dans le tableau I).

\section{Un test doit-il être plutôt sensible ou plutôt spécifique?}

Dans l'idéal, les deux... ! Cependant, les tests qui ont à la fois une sensibilité et une spécificité très élevées sont rares. On 
sera donc conduit à sélectionner un test dont les caractéristiques sont appropriées à la situation clinique.

Plus le test est sensible, moins il comportera de faux négatifs, et mieux il permettra, s'il est négatif, d'exclure la maladie : un moyen mnémotechnique de s'en souvenir est le vocable " $S_{n} O u t$ » en anglais, qui regroupe l'abréviation de la sensibilité ( $S n$ ») et le mot " out » qui désigne le fait d'exclure ("rule out») un diagnostic.

Plus le test est spécifique, moins il occasionnera de faux positifs, et mieux il permettra, s'il est positif, de confirmer la maladie. On peut se rappeler l'abréviation "SpIn », qui combine spécificité ( $\mathrm{Sp}$ ») et "rule in » ou confirmer un diagnostic.

Par exemple, dans des conditions de dépistage, on choisira de préférence un test très sensible, puisque l'objectif est la détection du plus grand nombre possible de sujets porteurs de la maladie. En revanche, on exigera du test de confirmation effectué chez les sujets dont le test de dépistage s'est révélé positif une spécificité très élevée, afin de minimiser le risque de faux positifs. De même, il est fréquent de devoir combiner plusieurs tests aux propriétés complémentaires dans une stratégie diagnostique.

Sensibilité et spécificité décrivent les caractéristiques d'un outil diagnostique et sont dérivées de populations de patients dont on connaît le statut de porteur ou non de la maladie M. Or, en pratique, le clinicien est confronté au résultat du test, mais ne sait évidemment pas si le patient a réellement la maladie recherchée ou non. Il doit donc connaître, si le test est positif, quelle est la probabilité que le patient soit réellement porteur de la maladie $\mathrm{M}$; et si le test est négatif, quelle est la probabilité que le patient ne soit pas porteur de la maladie M. Ces renseignements sont donnés par les notions de valeur prédictive positive et valeur prédictive négative d'un test.

\section{Valeur prédictive positive (VPP)}

La probabilité d'avoir la maladie $\mathrm{M}$ en cas de test positif s'appelle la valeur prédictive positive (VPP) d'un test. Elle est donnée par le rapport des vrais positifs sur l'ensemble des tests positifs. Pour le dosage du PNB (tableau III), sur 870 tests positifs, seuls 650 sont des vrais positifs, soit $75 \%$. Un patient dont le test est positif aura donc en réalité une probabilité de $75 \%$ d'avoir réellement une insuffisance cardiaque. Cette valeur est la probabilité post-test (ou a posteriori) d'avoir la maladie recherchée si le test est positif, une notion qui sera revue avec le théorème de Bayes [2].

\section{Valeur prédictive négative (VPN)}

La probabilité de ne pas souffrir de la maladie $M$ en cas de test négatif s'appelle la valeur prédictive négative (VPN) d'un test. Elle est donnée par le rapport des vrais négatifs sur l'ensemble des tests négatifs. Pour le dosage du PNB (tableau III), sur 668 tests négatifs, 596 sont des vrais négatifs, soit un rapport de $89 \%$. Un patient dont le test est négatif aura donc en réalité $89 \%$ de chance de ne pas souffrir d'insuffisance cardiaque. Par déduction, il aura donc $11 \%$ de risque de souffrir tout de même d'insuffisance cardiaque malgré un test négatif. Cette dernière valeur est la probabilité post-test (ou $a$ posteriori) d'avoir la maladie recherchée si le test est négatif, une notion qui sera également revue avec le théorème de Bayes [2].

\section{Conclusion}

On peut représenter de façon générique les notions vues jusqu'ici en construisant le tableau $I V$ [3]. En résumé, les notions de sensibilité et spécificité sont déterminées sur une population dont on connaît la présence ou l'absence de la maladie recherchée et représentent donc les caractéristiques intrinsèques d'un test donné. Les valeurs prédictives positive et négative, en revanche, fournissent la probabilité qu'un patient ait ou n'ait pas la maladie recherchée en fonction du résultat positif ou négatif du test. Nous verrons dans la note suivante [2] l'utilisation de ces notions en pratique pour évaluer comment change la probabilité initiale d'une maladie, une fois le résultat d'un test connu.

\section{Tableau IV.}

Résumé des notions de sensibilité, spécificité, valeur prédictive positive et valeur prédictive négative.

\begin{tabular}{|c|c|c|c|}
\hline \multirow[b]{2}{*}{$\begin{array}{l}\text { Résultat } \\
\text { du test }\end{array}$} & \multicolumn{2}{|c|}{ Test de référence } & \\
\hline & $\begin{array}{l}\text { Maladie } \\
\text { présente }\end{array}$ & $\begin{array}{l}\text { Maladie } \\
\text { absente }\end{array}$ & \\
\hline Test positif & $\begin{array}{l}\text { Vrais positifs } \\
\text { (a) }\end{array}$ & $\begin{array}{l}\text { Faux positifs } \\
\text { (b) }\end{array}$ & $\begin{array}{l}\text { Valeur prédictive } \\
\text { positive }=a /(a+b)\end{array}$ \\
\hline \multirow[t]{2}{*}{ Test négatif } & $\begin{array}{l}\text { Faux négatifs } \\
\text { (c) }\end{array}$ & $\begin{array}{l}\text { Vrais négatifs } \\
\text { (d) }\end{array}$ & $\begin{array}{l}\text { Valeur prédictive } \\
\text { négative }=d /(c+d)\end{array}$ \\
\hline & $\begin{array}{c}\text { Sensibilité }= \\
a /(a+c)\end{array}$ & $\begin{array}{l}\text { Spécificité = } \\
d /(b+d)\end{array}$ & $\begin{array}{c}\text { Prévalence } \\
\text { (probabilité } a \\
\text { priori })= \\
(a+c) /(a+b+c+d)\end{array}$ \\
\hline
\end{tabular}

Sensibilité : proportion de patients qui ont la maladie recherchée et dont le test est positif. Spécificité : proportion de patients qui n'ont pas la maladie recherchée et dont le test est négatif. Valeur prédictive positive : proportion des patients dont le test est positif et qui ont réellement la maladie recherchée. Valeur prédictive négative : proportion des patients dont le test est négatif et qui n'ont réellement pas la maladie recherchée. 


\section{Références}

1 McCullough PA, Nowak RM, McCord J, Hollander JE, Herrmann HC, Steg PG, Duc P, Westheim A, Omland T, Knudsen CW, Storrow AB, Abraham WT, Lamba S, Wu AH, Perez A, Clopton P, Krishnaswamy $\mathrm{P}$, Kazanegra R, Maisel AS : B-type natriuretic peptide and clinical judgment in emergency diagnosis of heart failure: analysis from
Breathing Not Properly (BNP) Multinational Study. Circulation 2002; $106: 416-22$.

2 Nendaz M, Perrier A: Theorème de Bayes et rapports de vraisemblance. Rev Mal Respir 2004 ; 21 : 394-7.

3 Sackett D, Haynes R, Guyatt G, Tugwell P : Clinical epidemiology. A basic science for clinical medicine. 2nd ed. Boston : Little, Brown and Co ; 1991. 